\title{
A Multi-Agent System for Organ Transplant Coordination
}

\author{
A. Aldea ${ }^{1}$, B. López ${ }^{2}$, A. Moreno ${ }^{1}$, D. Riaño ${ }^{1}$, A. Valls ${ }^{1}$ \\ ${ }^{1}$ Research Group on Artificial Intelligence \\ Departament d'Enginyeria Informàtica i Matemàtiques \\ Universitat Rovira i Virgili (URV) \\ Ctra.Salou s/n, 43006 Tarragona, Spain \\ \{aldea, amoreno, drianyo, avalls\}@etse.urv.es \\ ${ }^{2}$ Departament d'Electrònica, Informàtica i Automàtica \\ Universitat de Girona (UdG) \\ Campus Montilivi , 17071 Girona, Spain \\ blopez@silver.udg.es
}

\begin{abstract}
The coordination of human organ transplants is a difficult task that involves legal, clinical, organisational and human aspects within a complex distributed environment of hospitals and governmental institutions. We propose a Multi-Agent Architecture that is the kernel of an Intelligent Decision Support System to coordinate the Spanish activity in organ transplants. The different agents of the system are identified and the way they cooperate is explained in detail.
\end{abstract}

\section{Introduction}

There are two main reasons that justify the success of organ transplants in Spain. On one hand, new surgery techniques and medical treatments have been developed that increase the success of heart, pancreas, liver, kidney, and lung transplants [5]. On the other hand, modern societies have shown an important increase in the number of organs that are transplanted from a human brain-dead donor to a human living patient [2]. Whereas the first reason is related to the advances of Medicine, the second reason is related to the appearance of an organisational structure that coordinates all the stages of the donation and transplant process according to the local, regional, national, and international norms and laws. This structure and the idea of having a transplant coordinator for each hospital with a transplant unit defines the Spanish model [6], which is one of the most effective models in the world.

It is obvious that organising and coordinating transplants are complex tasks that require several clinical activities involving many people or working teams, and also an administrative process which is parallel to the clinical process. The main activities of the transplant process are [4]: detection of the potential organ donors, clinical examination of the organ donor, brain-death confirmation, maintenance and handling of the organ donor, legal brain-death confirmation, securing the family consent and the legal authorisation, arrangement of the organisational factors, organisation of the 
organ extraction and transplant, and clinical examination of the evolution of the receptor. Some of the people involved in these activities are: the patient who receives the organ, the physicians, the donor or dead patient, the family, the transplant coordinator, the nurses and the clinical staff, the extraction surgery team, the implantation surgery team, the staff of the laboratories, the legal advisors and the authorities, the logistic team, etc. These people must bring the administration tasks into line with the clinical tasks and follow the norms and legislation established by the healthcare authorities and organisations: the Organització Catalana de Transplantaments (OCATT) in Catalonia, the Organización Nacional de Trasplantes (ONT) in Spain, the United Network for Organ Sharing (UNOS) in the USA, the Eurotransplant International Foundation for some countries in the EU, the Canadian Association for Transplantation, etc.

Recent studies published by the ONT [2] show that the number of people who die while waiting for a heart, liver, or lung transplant in Europe fluctuates between the $15 \%$ and the $30 \%$. In order to improve the quality of public services, in the Information Society and Technology (IST) Program of the Fifth Framework Program, the European Commission proposes to foster the services and systems to the citizens in the area of HealthCare and Administration.

The ONT has identified six reasons that contribute to the loss of organ donations [2]: no detection, medical contraindications, family negatives, judicial negatives, maintenance losses, and failures during the surgical operation. Once these reasons have been identified, it is important to develop mechanisms to decrease the percentage of losses in each of the stages of the process. One way to improve the process is to elaborate an information system to be used as a decision support system in the coordination of transplants. This information system must have some communicative, informative and cognitive functions to guarantee a secure distributed communication, to maintain a historical file system, and to extract new knowledge from the system behaviour and from the analysis of the data.

In this work we present the architecture of a Multi-Agent System (MAS) that will support the communication and negotiation layers of the Transplant Coordination System that we have described in the above lines. This MAS is designed both to support the main activities of the transplant process, and also to be compatible with the current Spanish organisational structure. Moreover, the MAS is an open system that can be easily adapted to structural changes.

The rest of the paper is organised as follows. In section 2 we justify why a MAS is a good platform to ease the transplant coordination work that has been described in the introduction. In section 3 we supply an extended description of the proposed global MAS architecture. Section 4 describes the transplant coordination aspects. One of these aspects is the coordination of transplants from the point of view of a particular hospital. Section 5 describes a local MAS which is embedded in the global MAS and coordinates all the aspects that are local to a hospital. The paper ends with a discussion and the future work. 


\section{Applying Multi-Agent Systems to Organ Transplant Coordination: Justification and Motivation}

Health care authorities impose some structures in human organisations that cannot be violated, so maintaining them is essential [1][10]. The organisation of the transplant coordination in Spain is structured in four levels: national, zonal, regional and hospital level. At the hospital level all the data of patients is kept locally either centralised in a hospital patient clinical history warehouse or in the database of each hospital unit.

The type of data that the hospital keeps for each patient is quite diverse: electronic information such as clinical analysis results, images, signals, traditional X-ray photographs, ultrasounds and nurse recordings. The information of a patient is then spread in two dimensions: the type of support and the localisation. Current trends in hospital management are in the line of the automation of all the information related to a patient. In Spain information technologies have just started to be applied in major hospitals, although it will still take a long time to complete the automation of the whole history of patients. Automation opens the possibility to a centralised view of the data of patients. However, these data will still be localised and kept at the level of the hospital unit(s) where the patient has been treated. The reason is that every unit of the hospital is responsible of their patients and of the data stored about them. As a result, a distributed approach is quite appropriate to maintain the current existing organisational structure, as a centralised approach would break the authority and control of the activities of the hospitals and it would force a change in the information flow of the hospitals.

Multi-agent systems offer a platform for a distributed approach in which every agent is responsible of a given process, as units or hospitals are specialised on a given branch of Medicine. Moreover, agents can model other important tasks, as administrative tasks (gather information of a patient), logistics (how to address the problem of patient transport from a hospital to another hospital), etc. In addition, a MAS architecture allows the maintenance of the existent information systems by enhancing them to other possibilities through interface agents.

A MAS approach is particularly suitable for the organ transplant coordination problem as a great amount of information related to donor and receptor is required. All the necessary data may reside in different hospital units and also in different hospitals. Therefore, agents of different hospitals involved in a transplant process must cooperate to provide support to the transplant coordinator.

The benefit of such approach is twofold. On one hand, the application of MAS to organ transplant coordination allows the maintenance of the current organisational structure (either within a hospital or among different hospitals) and the use of the current information infrastructure of hospitals. On the other hand, the complexity of the application provides us with the opportunity to investigate a highly distributed problem with different levels of coordination and decision making. 


\section{General Architecture of the Multi-Agent System}

In order to coordinate organ transplants among Spanish hospitals we propose the construction of a MAS such as the one shown in figure 1. This architecture reflects the organ transplant coordination structure that is employed in Spain [7]. The national territory is partitioned into six zones. Each of these zones groups some of the nineteen Spanish autonomous regions.

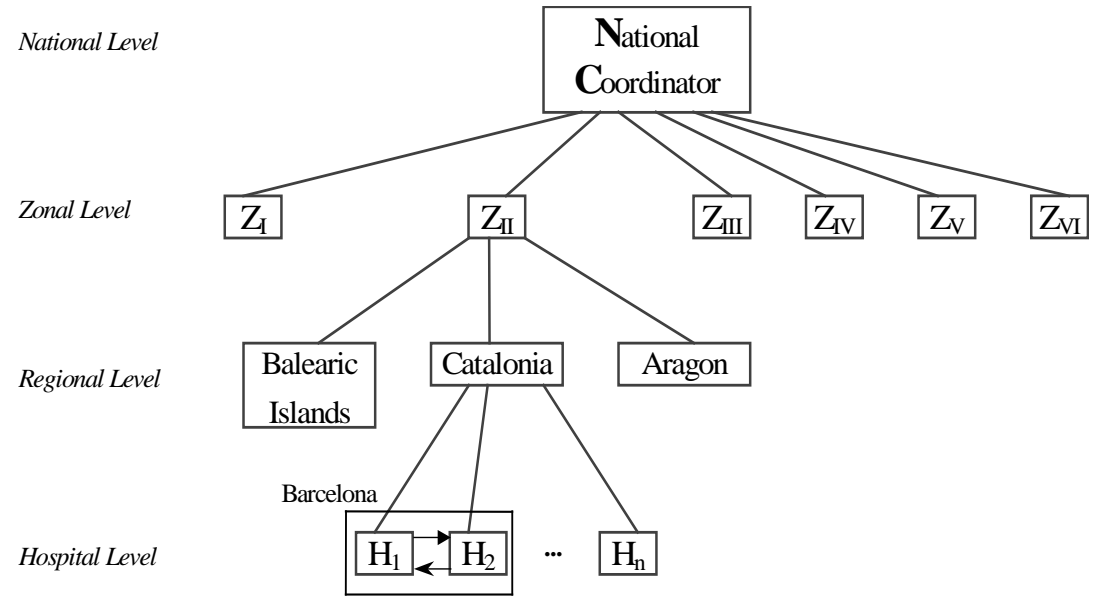

Fig. 1. Hierarchical dependencies among coordinators.

All these agents have different knowledge and play different roles, and they have to coordinate their activities to offer an efficient way of dealing with organ transplant management at the national level. There are two other agents in the global MAS, which have not been shown in figure 1 because they do not belong to the hierarchy:

- An agent called Emergency Coordinator (EC), which is the national coordinator of the 0 -emergency cases. This is the name given to those patients that are waiting for an organ and have reached a very critical condition, in which their life is at high risk if the transplant is not performed in a very short period of time.

- An agent called Historical Agent (HA), which receives the data of all transplants made in Spain. With this information it can keep historical files, elaborate statistics, apply data mining techniques in order to gain useful knowledge, etc.

It is important to stress the fact that, as argued in section 1, all the agents of the system must behave in accordance with the regulations imposed by regional and national transplant organisations, such as the Organització Catalana de Trasplantaments (OCATT) and the Spanish Organización Nacional de Trasplantes (ONT) [7]. 


\section{Transplant Coordination}

The coordination process that is followed when there is a new organ available for transplant in a hospital addresses in the first place the needs of those patients that have reached an extremely critical stage; if there are no such patients, it tries to find an appropriate recipient as close as possible to the donor's hospital, in order to minimise both the financial costs associated to the transport of the organ and the length of transport time. Note that, unlike other body components such as tissue or blood which can be frozen and stored, organs are available for a very short period of time. The implementation of each of the coordination steps is described in the following sections.

There are different occasions in the whole coordination process in which the donor's hospital $\left(\mathrm{H}_{\mathrm{i}}\right)$ receives a list of possible receptors of the organ. In those cases, $\mathrm{H}_{\mathrm{i}}$ performs the following process, which we call Search for the Most Appropriate Receptor of the Transplant (SMART). If the list is not empty, $\mathrm{H}_{\mathrm{i}}$ will start a reasoning process to choose the most appropriate patient, according to the characteristics of the organ and the potential receptors. This process can involve the use of an standard rule-based expert system, to seek advice from the human's hospital transplant coordinator, the performance of more detailed tests on the available organ, the use of case-based reasoning over the past history of transplants performed by $\mathrm{H}_{\mathrm{i}}$ and other hospitals, etc. Having found the best potential receptor, $\mathrm{H}_{\mathrm{i}}$ sends a message to its hospital $\left(\mathrm{H}_{\mathrm{j}}\right)$ with the detailed characteristics of the organ and the time limits on the transplant operation. $\mathrm{H}_{\mathrm{j}}$ will answer to $\mathrm{H}_{\mathrm{i}}$ whether it may use the organ or not (e.g. the potential recipient's hospital could lack the resources -operating theatres, especialised personnel- to perform the operation with those particular time constraints). If the answer is positive, then agent $\mathrm{H}_{\mathrm{i}}$ will inform $\mathrm{HA}$ so that it can update its records. At that point, the human transplant coordinators of $\mathrm{H}_{\mathrm{i}}$ and $\mathrm{H}_{\mathrm{j}}$ should get in touch and coordinate other activities such as the logistics of the transport of the organ from $\mathrm{H}_{\mathrm{i}}$ to $\mathrm{H}_{\mathrm{j}}$ and the management of the hospital staff so that everything is ready at $\mathrm{H}_{\mathrm{j}}$ to perform the transplant when the organ arrives. If $\mathrm{H}_{\mathrm{j}}$ answers that it cannot use the available organ, $\mathrm{H}_{\mathrm{i}}$ would repeat the same process with the following patient of the list. If none of the members of the list may receive the organ, the search for a receptor proceeds with the next step.

\subsection{Search 0-Emergency Patients}

Once an organ has been donated, the first step in the search for an appropriate receptor is to check whether there is any 0-emergency patient that may use the available organ. The agent that represents the hospital that has the new organ $\left(\mathrm{H}_{\mathrm{i}}\right)$ sends a message to EC with the information about the characteristics of the organ, so that EC may check whether there is any 0-emergency patient in all Spain that is waiting for an organ with similar characteristics. EC keeps a database with all the national 0-emergency patients, that it maintains with the updates (additions and deletions) that are sent daily from the hospital agents. EC would use some reasoning processes (e.g. it could use a rule-based expert system) in order to deduce which patients in critical state could use the organ. After that inference process, EC returns 
to $\mathrm{H}_{\mathrm{i}}$ a (possibly empty) list with all the 0-emergency cases that, in principle, could take advantage of the available organ. At that moment $\mathrm{H}_{\mathrm{i}}$ applies the SMART process outlined above to process this list of potential receptors.

If the list given by EC is empty, or if none of the urgent patients can actually use the organ, then it is time to search for a patient in the same hospital where the organ has been obtained, $\mathrm{H}_{\mathrm{i}}$, as detailed in the following section.

\subsection{Search Patients at the Same Hospital}

The next step is to check whether the organ may be used in the donor's hospital. Each hospital has a (possibly distributed) information system with the information of the local patients that are waiting for an organ. The detection and selection of the patients that can take advantage of a given organ is performed in a MAS which is local to the hospital, as is explained in section 5; in a nutshell, the hospital's transplant coordinator agent (TC) will try to find out whether there is any local patient that is compatible with the available organ by requesting this information to an agent that controls the access to the local databases of waiting patients (the database wrapper, WR). If the system is unable to find an appropriate patient in the same hospital, then it moves on to the next stage, in which the organ's receiver is searched in the hospitals of the same city.

\subsection{Search Patients at Other Hospitals of the Same City}

Transplant coordination at the city level is performed through direct communication between the hospitals of the city, as sketched in figure 2. The meaning of the four agents (IN, WR, OT and TC) that compose each local multi-agent system is explained with detail in section 5; suffice it to say that TC is the local transplant coordinator of a given hospital, who is responsible of the communication from the local multi-agent system to the national coordination system.

In the Spanish case, there are very few cities that have more than one hospital with transplant activities; therefore, there will be many times in which this step will be skipped. Each hospital knows which (if any) are the other hospitals of the same city, and it coordinates with them using the well-known Contract Net protocol [8][9]. Following this protocol, $\mathrm{H}_{\mathrm{i}}$ would send to the other hospitals the information of the available organ. They would check in their local databases whether they have any patient whose characteristics match those of the organ, and they would answer to $\mathrm{H}_{\mathrm{i}}$ the data of the patient or a refusal to use the organ. $\mathrm{H}_{\mathrm{i}}$ would receive different positive answers from different hospitals, and then it would follow the SMART process to get in touch with them and try to assign the available organ to one of them. If none of the hospitals of the same city may use the organ, $\mathrm{H}_{\mathrm{i}}$ will get in touch with the coordinator of its autonomous region to initiate the search for an appropriate patient outside the city. 


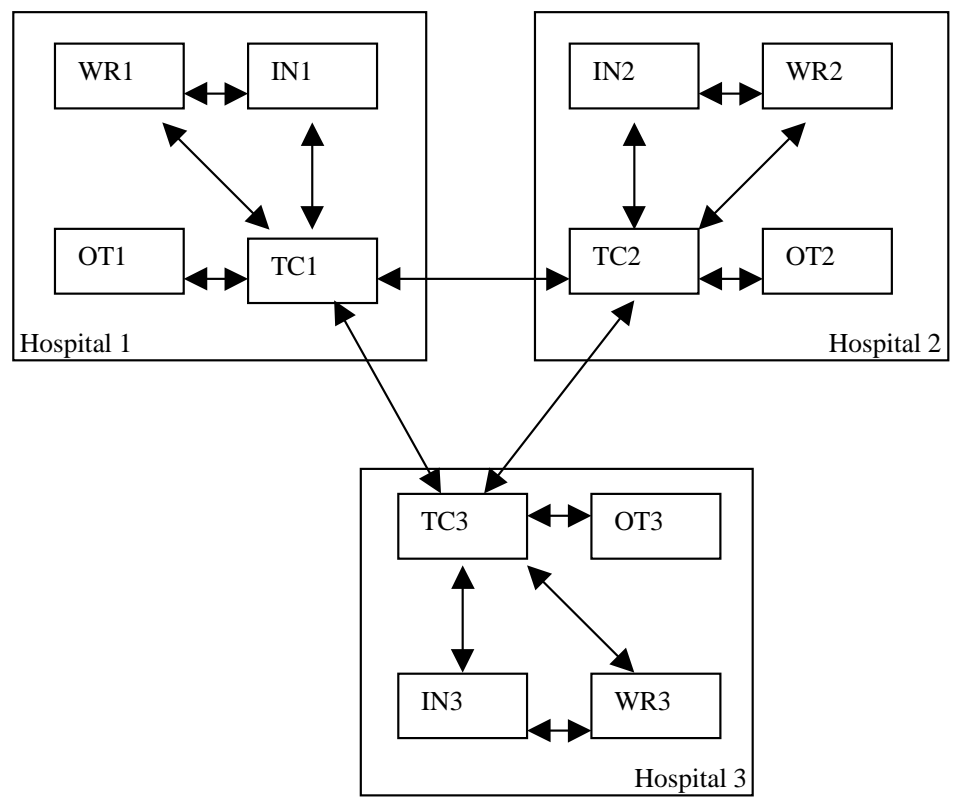

Fig. 2. Direct communication between the transplant coordinators of three hospitals of the same city

\subsection{Search Patients from Other Cities of the Same Region and Other Regions of the Same Zone}

The search of a patient in other cities is made in three different levels, that correspond to the levels of the hierarchy shown in figure 1: regional, zonal, and national level. The first two levels are covered in this section, whereas the national level is considered in section 4.5. First, the system looks for patients of the same region. $\mathrm{H}_{\mathrm{i}}$ sends the data of the organ to the coordinator of its autonomous region, $\mathrm{C}_{\mathrm{i}}$. This agent forwards this information to all the hospitals of the region that are not located in the same city than $\mathrm{H}_{\mathrm{i}}$. If several hospitals answer with the data of possible receivers of the organ, $\mathrm{C}_{\mathrm{i}}$ will send these data to $\mathrm{H}_{\mathrm{i}}$ so that it can get directly in touch with them, following again the SMART process. If there is not any positive answer from any hospital of the region, or $\mathrm{H}_{\mathrm{i}}$ considers that the potential candidates do not match the organ's characteristics, it will inform of this fact to its regional coordinator $\mathrm{C}_{\mathrm{i}}$ so that it can get in touch with its zone coordinator $\mathrm{Z}_{\mathrm{i}}$ (climbing up the hierarchy shown in figure 1 ).

$\mathrm{Z}_{\mathrm{i}}$ sends the organ information to the other regional coordinators of the same zone, and they, in turn, send it to the hospitals of their area, collect the positive answers and pass them along to $Z_{\mathrm{i}}$. $Z_{\mathrm{i}}$ has an ordered list of the hospitals of its zone. This list is updated as follows: each time that a hospital transplants an organ that came from the same zone but from a different city, it is moved to the last position of the list 
(this protocol has been established by ONT, [7]). The answers that $\mathrm{Z}_{\mathrm{i}}$ receives are ordered using the ranking induced by the position of the hospital in the list. The ordered list of patients is sent to $\mathrm{Ci}$, who passes it along to $\mathrm{H}_{\mathrm{i}}$, which gets in touch with the hospital $\mathrm{H}_{\mathrm{j}}$ of the patient in the first position, following the SMART process. When $\mathrm{H}_{\mathrm{i}}$ reaches an agreement with another hospital to send the organ, $\mathrm{Z}_{\mathrm{i}}$ will be informed so that it can update its ordered list of hospitals. If there is no appropriate patient to receive the organ, a petition is sent to the national level coordinator (NC) through the regional and zonal coordinators, $\mathrm{C}_{\mathrm{i}}$ and $\mathrm{Z}_{\mathrm{i}}$.

\subsection{Search Patients from Other National Zones}

The national coordinator, NC, receives the organ's characteristics from the donor's hospital, through the regional and zonal coordinators. This national agent maintains an ordered list of zones. When there is an hospital of a given zone that makes a transplant with an organ that came from a different zone, it is moved to the back of the list (this protocol has also been established by ONT, [7]). When NC receives the data of an organ, it tries to find receptors in the different zones in an ordered way, following the ranking induced by this list. NC sends the data of the organ to be transplanted to a zone coordinator, who sends it to the regional coordinators so that they can forward it to the hospitals. Each regional coordinator receives the answers from the different hospitals, and sends a list with all the possible receptors to the zone coordinator. This agent orders the lists of the different regions according to the ordered list of hospitals of the zone, and sends the result to NC who sends it, via the zonal and regional coordinator, to the donor's hospital (where the phase of direct contact between hospitals described in the SMART process would again take place). $\mathrm{NC}$ would repeat this process (following the ordered list of national zones) with all the zones, until it finds a hospital in which there is an appropriate receptor.

\section{Intra-Hospital Multi-Agent System}

Each hospital has a local information system with the information of the local patients that are waiting for an organ transplant. We propose the construction of a MAS in each hospital that can link these data with the national coordination transplant process that has been described in the previous section. The proposed architecture is shown in figure 3 . 


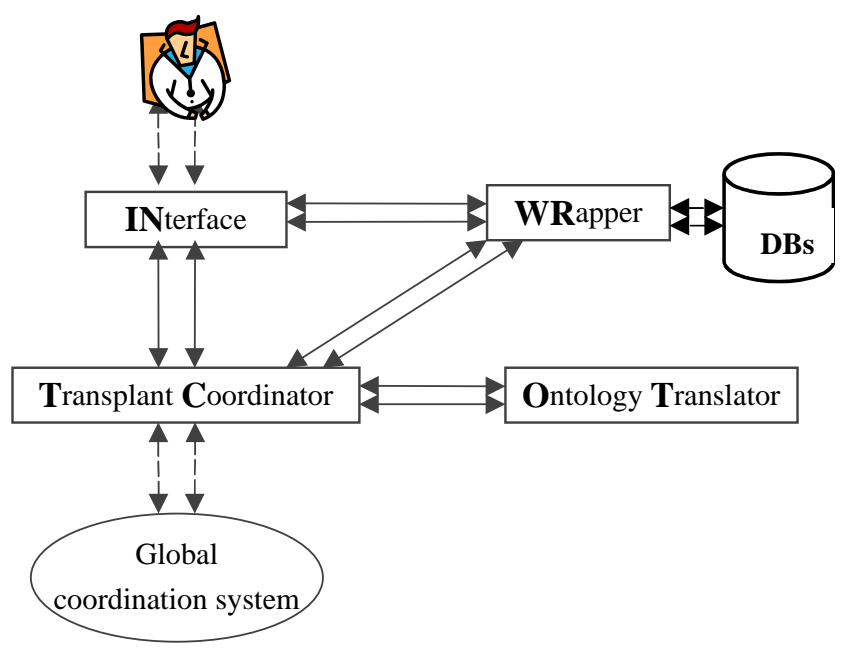

Fig. 3. Multi-agent local architecture

This figure shows that there are four agents in each hospital: the interface agent (IN), the hospital transplant coordinator (TC), the database wrapper (WR) and the ontology translator (OT). The goal of the interface agent is to manage the communication between the whole coordination system and the hospital staff. It provides an appropriate interface so that the hospital personnel may perform operations on the local patients' databases (additions, removals, modifications, queries) and introduce data of new available organs. The hospital transplant coordinator receives from agent IN the information about the new organs that are available at the hospital. At that moment the search for a possible receptor described in section 4 is initiated. As the medical ontologies used in each hospital are independent of each other, TC will ask the ontology translator (OT) to translate the data to an agreed common ontology before sending them to other agents, and to translate back the data received from other hospitals to the local ontology. The database wrapper receives messages from IN and TC with requests to perform operations on the hospital databases (updates and queries). It performs these operations on the databases, translating the queries into the database query language, executing them and sending back the obtained results.

\section{Discussion and Future Work}

In this paper we have presented the design of a multi-agent architecture for organ transplant coordination. The proposed architecture maintains the current Spanish organisational structures and informs about the performance of the overall system by employing an agent that keeps up to date the history of the transplant procedures. The automation of the coordination tasks allows the acceleration of the organ transplant process. 
Currently there are several research groups investigating the use of multi-agent systems for hospital management (see for instance [1]). However, this approach is different to ours as their main goal is to create a more profitable hospital system by decreasing the time that a patient stays in hospital and increasing the number of external patients.

The architecture presented in this paper focuses on the organ transplant coordination task. Two other research aspects are the reasoning mechanism behind the decision making process for each agent and the incorporation of learning methods. Works on decisions with multiple criteria as the ones in [3] will be applied for decision making. The historical information gathered by HA will be used as input to learning methods that will improve the MAS and the coordination process itself.

\section{References}

1. Decker, K., Li, J. Coordinated Hospital Patient Scheduling. Proc. ICMAS'98.

2. Informes y documentos de consenso. Organización Nacional de Trasplantes (Ed.), 2000.

3. Keeney R.L., Raiffa, H. Decision with Multiple Objectives: Preferences and Value Tradeoffs. Cambridge University Press, 1993.

4. López-Navidad, A., Domingo, P., Viedma, M.A., Professional characteristics of the transplant coordinator. Transplantation Proceedings, 29, pp. 1607-1613, Elsevier Science Inc., 1997.

5. López-Navidad, A., Kulisevsky, J., Caballero, F., El donante de órganos y tejidos. Evaluación y manejo. Springer-Verlag Ibérica, 1997.

6. Matesanz, R., Miranda, B., Coordinación y Trasplantes: El modelo español. Editorial Aula Médica, ISBN 84-7885-060-0, 1995.

7. Organización Nacional de Trasplantes, ONT: http://www.msc.es/ont.

8. Randall Davis, Reid G. Smith, Negotiations as a Metaphor for Distributed Problem Solving, Artificial Intelligence, Vol. 20, No. 1, pp. 63-109, January 1983.

9. Reid G. Smith, The Contract Net Protocol: High Load Communication and Control in a Distributed Problem Solver. IEEE Transactions on Computers, Vol. C-29, No. 12, pp. 11041113, December 1980.

10. So, Y., Durfee, E.H. Designing Organisation for Computational Agents. In: Simulating Organisations: Computational Models of Institutions and Groups. Edited by M.J. Prietula, K.M. Carly, L. Gasser. AAAI/Press/The MIT Press, 1998. 